\title{
A Importância do Natal nos Destinos de Gramado (Brasil) e Rovaniemi (Finlândia): um estudo a partir da percepção de Stakeholders ${ }^{I}$
}

\author{
The Importance of Christmas in Gramado (Brazil) and Rovaniemi (Finland) \\ Destinations: a study from the perception of stakeholders
}

\section{La Importancia de la Navidad en los Destinos de Gramado (Brasil) y Rovaniemi (Finlandia): un estudio de la percepción de stakeholders.}

\author{
Yoná da Silva Dalonso ${ }^{2}$ \\ Júlia Maria Lourenço ${ }^{3}$ \\ Paula Cristina Remoaldo ${ }^{4}$
}

\begin{abstract}
Resumo
Partindo do princípio de que o turismo é uma atividade que envolve um amplo grupo de atores, este artigo propõe a análise da percepção de stakeholders dos destinos de Gramado (Brasil) e de Rovaniemi (Finlândia), em relação ao turismo de cada cidade, especialmente no que se relaciona à principal atração dos destinos, o Natal. Para tanto, adota-se como metodologia de pesquisa a entrevista estandardizada, aplicada à atores ligados direta e indiretamente com a atividade turística dos destinos. Como resultado da pesquisa,observa-se a importância assumida pelo turismo, em especial do atrativo Natal, para o desenvolvimento dos destinos pesquisados, traduzida nas políticas públicas instituídas nos destinos e na inter-relação estabelecida entre os diferentes atores para a consolidação da marca "Natal" em cada destino.
\end{abstract}

Palavra-chave: Turismo; Natal; Stakeholders; Políticas públicas; Marca turística.

\begin{abstract}
Accepting that tourism is an activity that involves a wide group of stakeholders, this paper proposes the analysis of the perception of stakeholders from the destinations of Gramado (Brazil) and Rovaniemi (Finland) related to tourism in each city, especially related to
\end{abstract}

\footnotetext{
1 Trabalho apresentado no Fórum ABRATUR-15 realizado de 08 a 10 de junho de 2015 na Escola de Artes, Ciências e Humanidades da Universidade de São Paulo e indicado como melhor trabalho do evento.

${ }^{2}$ Doutora em Geografia, linha de pesquisa em Geografia e Planejamento Regional pela Universidade do Minho, Braga, Portugal. Professora e pesquisadora da Universidade da Região de Joinville, Santa Catarina. Brasil. E-mail: yona.dalonso@univille.br

3 Doutora em Engenharia Civil pela Universidade Técnica de Lisboa, Portugal. Professora Auxiliar do Departamento de Engenharia Civil e investigadora do Centro Território, Ambiente e Construção da Escola de Engenharia da Universidade do Minho, Braga, Portugal. E-mail: jloure@ civil.uminho.pt

${ }^{4}$ Doutora em Geografia Humana pela Universidade de Minho, Braga, Portugal. Professora Associada do Departamento de Geografia e Diretora do Doutoramento em Geografia e Co-Coordenadora do Grupo de Investigação em Espaço e Representação (SpaceR) do Laboratório de Paisagens, Património e Território (Lab2PT) da Universidade do Minho, Portugal. E-mail: cris.remoaldo@gmail.com
} 
Christmas attraction. The research methodology consists of a standard interview applied to actors directly and indirectly linked to the tourist activity in the destinations. As a result of the research, it is observed the importance of tourism, especially Christmas related, for the development of the researched destinations. This success is closely related to the implementation of the public policies for the consolidation of the "Christmas" brand in both destinations and the inter-relationships established between the different stakeholders.

Keywords: Tourism; Christmas; Stakeholders; Public policies; Tourist brand.

\section{Resumen}

Suponiendo que el turismo es una actividad que implica un amplio grupo de actores, el presente trabajo propone el análisis de la percepción de las partes interesadas de los destinos de Gramado (Brasil) y Rovaniemi (Finlandia), en relación con el turismo, especialmente en la principal atracción delos destinos, la Navidad. Así, se adopta la entrevista estandarizada como metodología de investigación, aplicada a los actores conectados directa y indirectamente con el turismo. Como resultado de la investigación, está la importancia adquirida por el turismo, especialmente la atracción de la Navidad para el desarrollo de los destinos, traducido en las políticas públicas establecidas y en las interrelaciónes entre los diferentes actores para la consolidación de la marca "Navidad" en cada destino.

Palabra clave: Turismo; Navidad; Stakeholders; Política pública; Marca turística.

\section{Introdução}

Para que o turismo seja sustentável, esforços no campo do planejamento e implementação de políticas devem ser eficazes, onde o sucesso desses esforços depende da cooperação dos muitos atores. Isto deve-se à natureza global, multi-sectorial do turismo e às complexidades sóciopolíticas envolvidas (Burns, 2004). Consequentemente, uma abordagem orientada para a comunidade, que enfatiza o papel que a comunidade desempenha na experiência turística vem, cada vez mais, sendo adotada (Getz, 2007).

A cooperação com a comunidade é um requisito fundamental para o planejamento sustentável e para o desenvolvimento dos destinos (BERITELLI, 2011, MALEK e COSTA, 2014), para a promoção e realização de projetos (VERNO et al., 2005), e para a criação e funcionamento no destino de estruturas de governança (YÜKSEL et al., 2005). Neste contexto, as comunidades compreendem uma variedade destakeholders que se interligam através de redes de atores de instituições públicas e privadas (DREDGE, 2006), e devem encontrar, mesmo que parcialmente, um consenso (BERITELLI, 2011). 
No estudo desenvolvido por Bramwell e Sharman (1999), são apresentados alguns benefícios potenciais quando os stakeholders colaboram na construção de um consenso em relação às políticas de turismo de um determinado destino. Em primeiro lugar, tal colaboração resolve muitos conflitos que podem surgir, a longo prazo, entre os stakeholders (HEALEY 1998). Em segundo lugar, a cooperação entre os públicos envolvidos pode ser mais legítima em termos políticos (BENVENISTE, 1989). Em terceiro lugar, esta colaboração melhora a coordenação de políticas e ações e promove a consciência em relação aos impactos econômicos, ambientais e sociais do turismo.

A colaboração das partes interessadas (stakeholders) no processo de desenvolvimento do turismo num determinado destino resolve alguns dos problemas relacionados com a falta de objetivos comuns (HALL, 2000; FRIEDMANN e MILES, 2002). Portanto, é importante que os planejadores do turismo reconheçam esses diversos grupos stakeholders e incorporem a perspetiva de que existem múltiplos interesses não são exclusivamente focados nas atividades de turismo.

Assim, a realidade dos territórios que também se desenvolvem como destinos turísticos apresenta um alto nível de complexidade e importa compreender os papéis desempenhados pelos diferentes stakeholders em presença (SAUTTER, LEISEN, 1999; WALIGO et al., 2013). A partir destes pressupostos, o presenta artigo apresenta a análise feita a partir de stakeholders das cidades de Gramado (Brasil) e Rovaniemi (Finlândia) em relação à importância do turismo para ambos os destinos, especialmente em relação ao atrativo Natal. Para a realização da pesquisa, utilizou-se a técnica da entrevista estandardizada, aplicada à agentes locais e regionais das cidades de Gramado e de Rovaniemi. Como resultado, observou-se uma forte relação assumida pelo Natal para o desenvolvimento de ambos os destinos. A análise possibilitou identificar os fatores-chave para a consolidação da marca "Natal" em ambos os destinos, a partir da percepção dos stakeholders selecionados para a pesquisa.

\section{O Natal e sua Influência para o Turismo dos Destinos de Gramado e Rovaniemi}

\subsection{Gramado}

A cidade brasileira de Gramado é um destino que produz as suas próprias políticas públicas, começando com a criação e consolidação de eventos, com base no "Natal Luz". Com uma população estimada em 34 mil habitantes (IBGE, 2013), a cidade é o principal destino turístico 
da Região das Hortênsias. Por se caracterizar como uma das cidades localizadas na região mais fria do país, Gramado é o principal destino turístico de inverno do Brasil.

Em 2012, a cidade recebeu 5,7 milhões de turistas, o que equivale a um aumento de aproximadamente 17\% em comparação com o ano anterior (SECRETARIA DE TURISMO DE GRAMADO, 2013).

O município é considerado um dos dez principais destinos de eventos do Brasil também devido às infraestruturas para eventos instaladas na cidade. De entre uma série de eventos realizados no destino, o evento Natal Luz reforça as características de cidade "europeia" e resgata a tradição natalina, trazida pelos imigrantes alemães. Desde o seu aparecimento, em 1986, é possível observar inovações na programação em cada edição do evento, consagrando-se, assim, como o maior evento natalino do Brasil (DALONSO et al., 2012).

No que se refere às políticas públicas para o turismo, Gramado constitui-se como um dos destinos indutores para o desenvolvimento do turismo regional, estabelecido no Programa de Regionalização do Turismo, do Ministério do Turismo do Brasil (Ministério do Turismo, 2009). A partir da análise da dimensão organizacional do turismo de Gramado, liderado pelo evento Natal Luz, o destino tem revelado um alinhamento das políticas, tanto no âmbito local, regional como nacional.

\subsection{Rovaniemi}

A cidade de Rovaniemi (Finlândia) desenvolveu as suas políticas de desenvolvimento do setor do turismo em torno do Christmas tourism (TERVO et al., 2013).Rovaniemi é a capital e pólo comercial da Lapônia, localizada na Finlândia, próxima do Círculo Polar Ártico. Com cerca de 59 mil habitantes, é o $13^{\circ}$ município finlandês em população (CITY OF ROVANIEMI, 2014). Mais conhecida como a casa oficial do Papai Noel (PRETES, 1995), esta região é considerada como sendo o segundo maior destino turístico da Finlândia, depois de Helsínquia, a capital finlandesa. Embora a Lapônia represente quase um terço do território do país, apenas $4 \%$ da população reside na região e a sua economia depende muito do turismo (HAAHTI e YAVAS, 2005; CITY OF ROVANIEMI, 2014).

Esta dependência econômica tornou-se mais forte depois de 1980, quando os planejadores da atividade iniciaram o desenvolvimento de novas estratégias de marketing, numa tentativa de rejuvenescer o turismo da Lapônia, já que as atrações existentes não eram suficientes. Em 1984, 
o Conselho Finlandês, em cooperação com as autoridades locais e regionais, começou um novo mercado turístico para a Lapônia denominado Santa Claus Land, iniciando-se, assim, a indústria de Natal da Finlândia (PRETES, 1995).

A "Vila do PapaiNoel" (tradução de Santa Claus Village) é apontada como a principal motivação turística para as pessoas visitarem Rovaniemi. Anualmente, cerca de 400 mil visitantes deslocam-se a Rovaniemi (CITY OF ROVANIEMI, 2014; TERVO et al., 2013).

O Christmas tourism em Rovaniemi tem mostrado um crescimento notável nos últimos anos. Apesar da principal atração ser o Papai Noel na época de Natal, os programas de Natal e as atividades relacionadas com o tema Natal desempenham um papel importante, quando se trata de atrair turistas para a cidade e região, em todos os períodos do ano.

De acordo com os objetivos e metas estratégicas da Região da Lapônia, acredita-se que a indústria do turismo, em especial do Christmas tourism, vai continuar a crescer nos próximos anos (LAPPI, 2011). Assim, Rovaniemi assume-se como o principal vetor turístico Região e um importante destino turístico da Finlândia, segundo o Ministério do Trabalho e da Economia da Finlândia, MEE (2014).

\section{Procedimentos de Pesquisa: a utilização das entrevistas estandardizadas}

O crescente desenvolvimento da investigação no turismo pode ser evidenciado a partir das inúmeras abordagens de pesquisa e do surgimento de novas técnicas utilizadas para investigar a atividade turística (COOPER, 2003).

Para Rejowski (1999), os aspectos metodológicos dos estudos em turismo podem ser abordados a partir de três formas:

a) visão reducionista: analisa detalhadamente o todo dentro do qual estão inseridos. O enfoque do estudo está nos elementos e não nas inter-relações;

b) visão holística: trata-se de uma visão que considera todas as partes interrelacionáveis, não são sendo passíveis de se analisar de forma isolada;

c) visão sistémica: surge em função das limitações das aproximações reducionista e holística. A análise do turismo é feita com base num sistema, que permite observar particularidades do todo e, ao mesmo tempo, propriedades específicas das partes que compõem esse todo. 
No turismo, as metodologias e os métodos adotados nas pesquisas não podem ser discutidos sem primeiramente se considerarem os seus significados e os seus paradigmas. Os paradigmas servem para informar os métodos utilizados nos estudos em turismo, assim como, de forma geral, nas ciências sociais (JENNINGS, 2009).Finn et al. (2000) categorizam as investigações em turismo em três grupos: investigação teórica (sem evidência empírica), investigação empírica (sem teoria) e estudos descritivos.

No âmbito da pesquisa feita para o presente trabalho, recorreu-se à aplicação da entrevista estandardizada ou diretiva com personalidades ligadas direta e indiretamente com a atividade turística dos destinos de Gramado e de Rovaniemi. A escolha dos destinos deu-se em função do atrativo "Natal", respetivamente denominados"Natal Luz" e "Natal na Lapônia" (tradução de Christmas in Lapland) exercerem um papel determinante, nas últimas décadas, na transformação do turismo como principal atividade econômica de toda a região em que se inserem.

Segundo Cohen et al. (2013), a entrevista estandardizada possibilita uma maior comparabilidade das respostas, já que os respondentes respondem às mesmas perguntas, além de que reduz os efeitos e a influência do entrevistador quando são feitas várias entrevistas. $\mathrm{O}$ método, da mesma forma, facilita a organização e análise dos dados obtidos na entrevista. Para a estruturação das perguntas, foi estabelecido um conjunto de temas que permitiram dar alguma coerência e homogeneidade às diferentes entrevistas concretizadas (Quadro 1).

Quadro 1: Temas das entrevistas realizadas a vários stakeholders

\begin{tabular}{|c|c|c|}
\hline Tema & Título & Perguntas \\
\hline 1 & $\begin{array}{l}\text { Importância do turismo } \\
\text { para a cidade (impactos) }\end{array}$ & $\begin{array}{l}\text { a) Na sua perspetiva, qual é a importância do turismo para } \\
\text { Gramado/Rovaniemi? } \\
\text { Setor ecónômico: mais importante /um dos mais importantes/ um } \\
\text { dos vários existentes } \\
\text { Qual é a importância do Natal/Santa Claus no conjunto do } \\
\text { turismo para Gramado/Rovaniemi? } \\
\text { Segmento turístico: mais importante /um dos mais importantes/ } \\
\text { um dos vários existentes } \\
\text { Quais são os outros segmentos / eventos igualmente importantes } \\
\text { ou com importância significativa? } \\
\text { b) Na sua opinião, quais foram os impactos desencadeados pelo } \\
\text { turismo em Gramado/Rovaniemi? (positivos e/ou negativos) } \\
\text { d) Na sua opinião, quando se pode afirmar que efetivamente se } \\
\text { iniciou o turismo em Gramado/Rovaniemi? }\end{array}$ \\
\hline 2 & $\begin{array}{l}\text { Inserção regional do } \\
\text { destino }\end{array}$ & $\begin{array}{l}\text { c) O destino de Gramado/Rovaniemi é o principal responsável } \\
\text { pelo desenvolvimento do turismo na região em que se insere? } \\
\text { f) Como se dá a inserção regional de Gramado/Rovaniemi? }\end{array}$ \\
\hline 3 & $\begin{array}{l}\text { Participação ativa nas } \\
\text { políticas de turismo } \\
\text { (locais e regionais) }\end{array}$ & $\begin{array}{l}\text { f) A elaboração de políticas públicas para o setor do turismo } \\
\text { passa por uma discussão e participação da comunidade local? } \\
\text { Quais foram as mais importantes ao longo do tempo? }\end{array}$ \\
\hline
\end{tabular}




\begin{tabular}{|l|l|l|}
\hline 4 & $\begin{array}{l}\text { Ações de marketing e } \\
\text { promoção do destino }\end{array}$ & $\begin{array}{l}\text { m) Como avalia a estratégia de marketing externo do destino } \\
\text { Gramado/Rovaniemi? }\end{array}$ \\
\hline 5 & $\begin{array}{l}\text { Integração com a } \\
\text { comunidade residente } \\
\text { (participação da } \\
\text { sociedade civil/atores) }\end{array}$ & $\begin{array}{l}\text { g) Quais são os públicos que mais beneficiam com o turismo em } \\
\text { Gramado/Rovaniemi? No percurso da atividade estes foram } \\
\text { sempre os públicos que beneficiaram mais? } \\
\text { h) Na sua opinião, que tipo de interação existe entre o Poder } \\
\text { Público, as Organizações e a Sociedade Civil? Como foi esta } \\
\text { interação no início do turismo no destino? }\end{array}$ \\
\hline 6 & $\begin{array}{l}\text { Interação com os } \\
\text { visitantes }\end{array}$ & $\begin{array}{l}\text { i) Como avalia a interação entre os turistas e os residentes? } \\
\text { Sempre se deu desta forma? } \\
\text { j) Considera que o turismo é bem aceite na cidade? Foi sempre } \\
\text { assim desde o início? }\end{array}$ \\
\hline 7 & $\begin{array}{l}\text { Caracterização dos } \\
\text { negócios de turismo }\end{array}$ & $\begin{array}{l}\text { no turismo de Gramado/Rovaniemi e em que épocas foram feitos? } \\
\text { 1) Atualmente, que tipo de negócio é prioritário para o turismo de } \\
\text { Gramado/Rovaniemi? A partir de quando foi estabelecido como } \\
\text { prioridade? }\end{array}$ \\
\hline 8 & Ameaças ao destino & $\begin{array}{l}\text { n) Na sua opinião, o Natal sofreu mudanças significativas ao } \\
\text { longo do tempo? Mudanças positivas ou negativas? } \\
\text { o) Quais são as principais ameaças para o turismo de } \\
\text { Gramado/Rovaniemi? }\end{array}$ \\
\hline 9 & $\begin{array}{l}\text { Expectativas futuras para } \\
\text { puais são as suas expectativas futuras em relação ao turismo } \\
\text { em Gramado/Rovaniemi? }\end{array}$ \\
\hline Furismo no destino & 2015). \\
\hline
\end{tabular}

Fonte: elaborado a partir de Dalonso (2015).

Um dos critérios seguidos para a seleção dos entrevistados foi o de garantir a presença de stakeholders ligados direta e indiretamente com a atividade turística de ambos destinos. Neste sentido, a amostra resultou na identificação de 4 entrevistados em Gramado (Quadro 2) e 5 entrevistados em Rovaniemi (Quadro 3).

Quadro 2: Lista de entrevistas realizadas com stakeholders de Gramado

\begin{tabular}{|l|l|}
\hline Identificação do Entrevistado & Função que desempenha \\
\hline Rosa Helena Folk & Secretária da Secretaria de Turismo de Gramado \\
\hline Rogério Poloni & Ex-Presidente da Convention \& Visitors Bureau Região das Hortênsias \\
\hline Marina Wöhlke Cyrillo & $\begin{array}{l}\text { Professora do Instituto Federal de Educação, Ciência e Tecnologia do Rio } \\
\text { Grande do Sul }\end{array}$ \\
\hline Geísa Schulze & Representante da comunidade de Gramado \\
\hline
\end{tabular}

Fonte: Elaborado a partir de Dalonso (2015).

Quadro 3: Lista de entrevistas realizadas com stakeholders de Rovaniemi

\begin{tabular}{|l|l|}
\hline Identificação dos stakeholders & Função que desempenha \\
\hline Anja Tuhoino & Pesquisadora da Universidade do Leste da Finlândia \\
\hline Sanna Kärkkäinen & Gestora de turismo da Rovaniemi Tourism \& Marketing Ltd \\
\hline Satu Luiro & Assessora de turismo do Conselho Regional da Lapônia \\
\hline Eila Linna & Pesquisadora da Universidade da Lapônia \\
\hline
\end{tabular}

Fonte: Elaborado a partir de Dalonso (2015).

A aplicação das entrevista e, consequentemente, o desenvolvimento do presente estudo teve como propósito discutir algumas das idéias em relação à configuração turística dos destinos, 
especialmente no que se relaciona ao Natal e a relevância assumida em relação ao processo de desenvolvimento de ambos os destinos. Neste sentido, apresentam-se na sequência a sistematização das entrevistas realizadas com os stakeholders selecionados dos destinos de Gramado e Rovaniemi.

\subsection{Importância do turismo para a cidade}

Inicia-sea análise das entrevistas com as respostas às perguntas relacionadas com o tema 1: importância do turismo para a cidade. Todos os entrevistados foram de opinião de que o turismo é de elevada importância para a cidade (Quadro 04).

Quadro 041: Tipo de importância do turismo para a cidade

\begin{tabular}{|c|c|c|}
\hline \multicolumn{2}{|c|}{ Entrevistados } & Categoria* \\
\hline \multirow{4}{*}{ 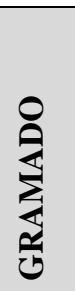 } & Rosa Helena Folk & 1 \\
\hline & Rogério Poloni & 1 \\
\hline & Marina Wöhlke Cyrillo & 3 \\
\hline & Geísa Schulze & 1 \\
\hline \multirow{4}{*}{ 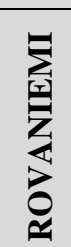 } & Anja Tuhoino & 1 \\
\hline & Sanna Kärkkäinen & 1 \\
\hline & Satu Luiro & 1 \\
\hline & Eila Linna & 1 \\
\hline
\end{tabular}

*Hierarquização da importância atribuída ao turismo para a cidade adotou-se a escala: 1- elevada importância / 2- pouca importância / 3- não respondeu de forma direta

Fonte: elaborado a partir de Dalonso (2015).

Em Gramado o turismo foi considerado por todos os entrevistados como uma atividade extremamente importante para a economia e o desenvolvimento da cidade de Gramado $(n=4)$. O motivo mais enfatizado pelos entrevistados em relação à importância do turismo para a cidade está relacionado com a dependência económica de Gramado em relação à atividade turística. Gramado é hoje o principal destino turístico do Rio Grande do Sul, onde toda a cidade se desenvolve em prol do turismo.

Em relação à importância do turismo para a cidade de Rovaniemi, todos os entrevistados $(n=4)$ consideraram esta atividade de elevada importância para a cidade. A atividade turística é avaliada como um importante fator de desenvolvimento para a cidade e para a Região. A atividade turística, iniciada a partir da Segunda Guerra Mundial e impulsionada a partir de 1970, 
é hoje vital para a população residente. Em relação aos impactes, todos entrevistados, da mesma forma, afirmam que são muito positivos para Rovaniemi.

\subsection{Inserção regional do destino}

Em relação ao tema 2 (Inserção regional do destino), foram tomadas em consideração as seguintes questões: 1) O destino (Gramado/Rovaniemi) é o principal responsável pelo desenvolvimento do turismo na região em que se insere? 2) Como se dá a inserção? O Quadro 05 apresenta o grau de hierarquização das respostas dos entrevistados..

Quadro 05: Inserção Regional do destino

\begin{tabular}{|c|c|c|}
\hline \multicolumn{2}{|r|}{ Entrevistados } & Categoria* \\
\hline \multirow{4}{*}{ 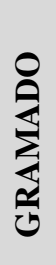 } & Rosa Helena Folk & 1 \\
\hline & Rogério Poloni & 1 \\
\hline & Marina Wöhlke Cyrillo & 2 \\
\hline & Geísa Schulze & 1 \\
\hline \multirow{4}{*}{ 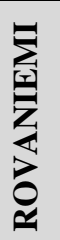 } & Anja Tuhoino & 1 \\
\hline & Sanna Kärkkäinen & 1 \\
\hline & Satu Luiro & 1 \\
\hline & Eila Linna & 1 \\
\hline
\end{tabular}

*1- elevada / 2- pouco elevada / 3- não respondeu de forma direta.

Fonte: elaborado a partir de Dalonso (2015).

Nas quatro entrevistas realizadas em Gramado, constatou-se que três entrevistados consideraram a inserção Regional de Gramado elevada e uma entrevistada considerou pouco elevada. Apesar de Gramado ser o mais importante destino da Região das Hortênsias, os outros destinos que integram a região também desempenham a função de catalisadores para o desenvolvimento regional do turismo.

Em relação à Rovaniemi, além do destino ser a maior cidade da região da Lapônia, a cidade desempenha um importante papel de articulador regional dado ser a sede administrativae o maior centro de estudos da região da Lapônia. Na avaliação de todos os entrevistados $(n=4)$, Rovaniemi desempenha uma função estratégica na interlocução com os demais municípios da região, assumindo a condição de destino-chave da Lapônia. Esta posição assumida implica que, na maioria das vezes, caiba a Rovaniemi o dispender de uma maior concentração de recursos e esforços em projetos de amplitude regional. 


\subsection{Participação ativa nas políticas de turismo (locais e regionais)}

$\mathrm{Na}$ análise das narrativas relacionadas com o tema 3 (Participação ativa nas políticas de turismo: locais e regionais), foi considerada a seguinte questão: a elaboração de políticas públicas para o setor do turismo passa por uma discussão e participação da comunidade local? Na mesma questão perguntou-se aos entrevistados se sabiam quais foram as mais importantes políticas públicas ao longo do tempo. O Quadro 06 apresenta a categorização da relevância dada ao tema.

Quadro 06: Participação ativa nas políticas de turismo (locais e regionais)

\begin{tabular}{|c|c|c|}
\hline \multicolumn{2}{|r|}{ Entrevistados } & Categoria* \\
\hline \multirow{4}{*}{ 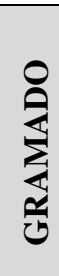 } & Rosa Helena Folk & 1 \\
\hline & Rogério Poloni & 2 \\
\hline & Marina Wöhlke Cyrillo & 3 \\
\hline & Geísa Schulze & 1 \\
\hline \multirow{4}{*}{ 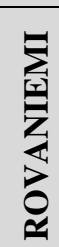 } & Anja Tuhoino & 1 \\
\hline & Sanna Kärkkäinen & 1 \\
\hline & Satu Luiro & 1 \\
\hline & Eila Linna & 1 \\
\hline
\end{tabular}

*1- elevada / 2- pouco elevada / 3- não respondeu de forma direta.

Fonte: elaborado a partir de Dalonso (2015).

$\mathrm{Na}$ análise das entrevistas de Gramado, evidenciou-se pontos de vista muito distintos. Dois dos quatro entrevistados apontaram para a existência de uma elevada participação da comunidade nas discussões em relação às políticas públicas de Gramado. Observou-se que esta interação da comunidade deu-se com mais intensidade no início do desenvolvimento turístico do destino. Entretanto, somente um dos entrevistados especificou quais as políticas que foram importantes ao longo do tempo.

Em relação à Rovaniemi, todas as entrevistadas $(n=4)$ avaliaram como elevada a participação da comunidade local na discussão em relação às políticas de turismo de Rovaniemi e da Lapônia. O Conselho Regional da Lapônia é responsável em coordenar as estratégias de desenvolvimento regional, o financiamento para o desenvolvimento de projetos, tanto nacionais quanto da União Européia, com uma forte participação da comunidade e da classe empresarial. 


\subsection{Ações de marketing e promoção}

Em relação às ações de marketing e promoção no destino, o Quadro 07 apresenta a categorização da opinião dos entrevistados entre positiva, pouco positiva e negativa, quando questionados sobre como avalia a estratégia de marketing externo dos destinos de Gramado e Rovaniemi.

Quadro 07:2Ações de marketing e promoção do destino

\begin{tabular}{|c|c|c|}
\hline \multicolumn{2}{|r|}{ Entrevistados } & Categoria* \\
\hline \multirow{4}{*}{ 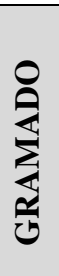 } & Rosa Helena Folk & 1 \\
\hline & Rogério Poloni & 2 \\
\hline & Marina Wöhlke Cyrillo & 1 \\
\hline & Geísa Schulze & 1 \\
\hline \multirow{4}{*}{ 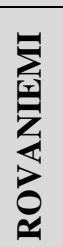 } & Anja Tuhoino & 1 \\
\hline & Sanna Kärkkäinen & 1 \\
\hline & Satu Luiro & 1 \\
\hline & Eila Linna & 1 \\
\hline
\end{tabular}

Fonte: elaborado a partir de Dalonso (2015).

A partir da análise das respostas dos entrevistados de Gramado, observou-se que as ações de marketing estão concentradas nos mercados brasileiros. Apesar de Gramado atrair turistas do Mercosul, foi percetível a necessidade de se promover ainda mais no Brasil, na América Latina e na Europa. Outro fator observado foi a dependência do destino dos roteiros dos operadores turísticos. A oferta de roteiros turísticos para Gramado apresenta-se como um forte produto nas agências e operadores turísticos do Brasil.

Nas respostas dos entrevistados de Rovaniemi é percetível o alinhamento existente entre as ações de marketingdo destino e a Região da Lapônia. Apesar do grande mercado consumidor se concentrar no próprio território europeu, mercados como a Rússia, a Grã-Bretanha, a China e o Japão são cada vez mais prioritários para a Lapônia. A marca do Papai Noel é considerada como a principal marca turística da Lapônia.

\subsection{Integração com a comunidade residente (participação da sociedade civil/atores)}

Em relação ao tema 5 (Integração com a comunidade residente e participação da sociedade civil/atores), foi realizada a análise a partir de duas questões colocadas aos entrevistados. 
Primeiramente, questionou-se quais são os públicos que mais beneficiam com o turismo e se no decorrer da atividade estes foram sempre os públicos que mais beneficiaram. Depois, foi perguntada a opinião em relação à interação que existe entre o poder público, as organizações e a sociedade civil, bem como se saberiam avaliar se esta interação se deu, no destino, desde o início da atividade turística. O Quadro 08 apresenta o grau de integração com a comunidade residente, avaliado pelos entrevistados.

Quadro 08: Integração com a comunidade residente (participação da sociedade civil/atores)

\begin{tabular}{|c|c|c|}
\hline \multicolumn{2}{|r|}{ Entrevistados } & Categoria* \\
\hline \multirow{4}{*}{ 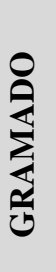 } & Rosa Helena Folk & 1 \\
\hline & Rogério Poloni & 2 \\
\hline & Marina Wöhlke Cyrillo & 3 \\
\hline & Geísa Schulze & 1 \\
\hline \multirow{4}{*}{ 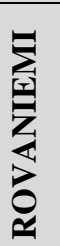 } & Anja Tuhoino & 3 \\
\hline & Sanna Kärkkäinen & 1 \\
\hline & Satu Luiro & 1 \\
\hline & Eila Linna & 1 \\
\hline
\end{tabular}

Em Gramado, apesar da representante da comunidade reconhecer a interação da comunidade residente com o poder público e os demais atores do turismo, opinião reafirmada pela Secretária de Turismo de Gramado, um dos entrevistados reconhece que esta interação já foi maior noutros períodos do desenvolvimento do turismo da cidade. Esta interação decorre da dependência económica dos residentes em relação à atividade turística, já que grande parte da comunidade trabalha para o turismo, incluindo os trabalhadores do setor industrial.

No que diz respeito à integração entre a comunidade residente em Rovaniemi, o poder público, as organizações e as empresas do turismo torna-se percetível um certo alinhamento entre estes públicos. Particularmente, o poder público, através da representação regional (Conselho Regional da Lapônia) e local (Rovaniemi Tourism \& Marketing Ltda) disponibiliza recursos e ferramentas para o cidadão que queira investir no turismo. A maioria dos entrevistados consideraram como positiva a integração existente $(n=3)$. Apesar de a pesquisadora Anja 
Tuhoino não identificar claramente a integração entre estes diferentes públicos, ela considera como importante o papel desempenhado pelo Governo na disponibilização de recursos.

\subsection{Interação com os visitantes}

Em relação ao tema 6 (Interação com os visitantes) foram levadas em consideração as duas seguintes perguntas (Quadro 09) : 1) Como avalia a interação entre os visitantes e os residentes? Sempre se deu desta forma?; 2) Considera que o turismo é bem aceite na cidade? Foi sempre assim desde o início?

Quadro 09: Interação com os visitantes

\begin{tabular}{|c|c|c|}
\hline \multicolumn{2}{|r|}{ Entrevistados } & Categoria* \\
\hline \multirow{4}{*}{ 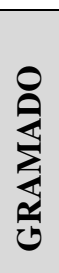 } & Rosa Helena Folk & 1 \\
\hline & Rogério Poloni & 1 \\
\hline & Marina Wöhlke Cyrillo & 2 \\
\hline & Geísa Schulze & 1 \\
\hline \multirow{4}{*}{ 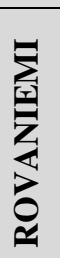 } & Anja Tuhoino & 1 \\
\hline & Sanna Kärkkäinen & 1 \\
\hline & Satu Luiro & 1 \\
\hline & Eila Linna & 1 \\
\hline
\end{tabular}

Fonte: elaborado a partir de Dalonso (2015).

$\mathrm{Na}$ categorização apresentada em relação ao grau de interação existente em Gramado, observou-se uma preocupação em relação ao lazer e à qualidade de vida da comunidade residente. Apesar de a maioria dos entrevistados considerar como positiva a interação entre o turista e a comunidade residente $(n=3)$, um dos pontos de vista exposto é o de haver uma certa artificialidade nas relações entre o turista e o residente.

Numa perspetiva mais positiva do destino de Rovaniemi, todos os entrevistados $(n=4)$ avaliaram que há uma boa relação entre os turistas e os residentes. Tal fator deve-se à dependência económica do turismo, observada na maioria das entrevistas realizadas $(n=3)$. Para os entrevistados, a cidade de Rovaniemi construiu uma identidade de bem-receber os turistas, caraterizando-se como sendo algo que ocorre naturalmente no destino. 


\subsection{Caracterização dos negócios de turismo}

Em relação ao tema 7 (caracterização dos negócios de turismo), faz-se a análise a partir das seguintes questões: 1) Na sua opinião, quais foram os principais investimentos feitos no turismo de Gramado e de Rovaniemi e em que épocas foram feitos? 2) Atualmente, que tipo de negócio é prioritário para o turismo de Gramado? A partir de quando foi estabelecido como prioridade? O Quadro 10 categoriza os investimentos.

Quadro 10: Caracterização dos negócios de turismo

\begin{tabular}{|c|c|c|}
\hline \multicolumn{2}{|r|}{ Entrevistados } & Categoria* \\
\hline \multirow{4}{*}{ 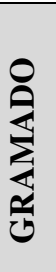 } & Rosa Helena Folk & 1 \\
\hline & Rogério Poloni & 1 \\
\hline & Marina Wöhlke Cyrillo & 2 \\
\hline & Geísa Schulze & 1 \\
\hline \multirow{4}{*}{ 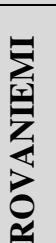 } & Anja Tuhoino & 1 \\
\hline & Sanna Kärkkäinen & 1 \\
\hline & Satu Luiro & 1 \\
\hline & Eila Linna & 1 \\
\hline
\end{tabular}
respondeu de forma direta.

Fonte: elaborado a partir de Dalonso (2015)

Em relação à Gramado, a maioria dos entrevistados $(n=3)$ consideraram que, ao longo dos anos, grandes e importantes investimentos foram realizados em Gramado. Grande parte destes investimentos relaciona-se com o evento Natal Luz e a consolidação da marca turística do destino, despertando o interesse em novos empreendimentos na cidade. Porém, os entrevistados identificaram a necessidade em se investir na expansão da capacidade hoteleira.

Quanto à Rovaniemi, todos os entrevistados $(n=4)$ consideram que o turismo a partir do atrativo Natal é um dos principais focos para o investimento turístico do destino. A partir da inauguração da Aldeia do Papai Noel (em 1984), Rovaniemi passou a contar com investimentos sucessivos na cidade e na região. É identificado um crescimento nos investimentos privados, especialmente na área da prestação de serviços. 


\subsection{Ameaças ao destino}

Em relação ao tema 8 (ameaças ao destino), foram colocadas as seguintes questões: 1) Quais são as principais ameaças para o turismo de Gramado e de Rovaniemi? 2) Na sua opinião, o Natal sofreu mudanças significativas ao longo do tempo? Mudanças positivas ou negativas? O Quadro 11 categoriza o grau de existência de ameaças no destino.

Quadro 11:Ameaças ao destino

\begin{tabular}{|c|c|c|}
\hline \multicolumn{2}{|r|}{ Entrevistados } & Categoria* \\
\hline \multirow{4}{*}{ } & Rosa Helena Folk & 1 \\
\hline & Rogério Poloni & 1 \\
\hline & Marina Wöhlke Cyrillo & 2 \\
\hline & Geísa Schulze & 1 \\
\hline \multirow{4}{*}{ 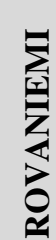 } & Anja Tuhoino & 1 \\
\hline & Sanna Kärkkäinen & 1 \\
\hline & Satu Luiro & 1 \\
\hline & Eila Linna & 1 \\
\hline
\end{tabular}

Uma das grandes preocupações apontadas pela maioria dos entrevistados de Gramado (n=3) é o crescimento contínuo do turismo no destino. Em relação à figura do Papai Noel, também foram ponderadas as mudanças ocorridas durante o desenvolvimento do Natal Luz na cidade e a sua importância para a economia de Gramado. O destino encontra-se numa fase de consolidação e o aumento no número de visitantes pode ser caracterizado como um fator de desequilíbrio para a qualidade do turismo de Gramado. Segundo o apurado durante as entrevistas, a necessidade de um maior investimento em infraestruturas de acesso, na expansão hoteleira e na gestão profissional do Natal Luz são ações estratégicas para o destino de Gramado.

Quanto à Rovaniemi, a acessibilidade e as mudanças climáticas foram consideradas como as principais ameaças ao destino. Em relação à acessibilidade, percebe-se, através das narrativas dos entrevistados, a necessidade de se ampliar o número de vôos e conexões para outros destinos da Europa, pretendendo, com isso, diminuir o tempo de deslocação até Rovaniemi. 
Relativamente às preocupações com as mudanças climáticas, o ponto crítico apontado pelos entrevistados é a dependência do destino em relação ao turismo de inverno, sendo, assim, a neve e o gelo os principais elementos de atração turística. Já em relação à figura do Papai Noel, percebem-se opiniões muito distintas quanto às mudanças observadas ao longo do tempo.

\subsection{Expectativas futuras para o turismo no destino}

Por fim, no tema 9 (expectativas futuras para o turismo no destino), foi colocada a seguinte questão aos entrevistados: Quais são as suas expectativas futuras em relação ao turismo em Gramado e em Rovaniemi? A análise da categorização estabelecida é apresentada no Quadro 12.

Quadro 12: Expectativas futuras para o turismo no destino

\begin{tabular}{|c|c|c|}
\hline \multicolumn{2}{|r|}{ Entrevistados } & Categoria \\
\hline \multirow{4}{*}{ 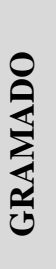 } & Rosa Helena Folk & 1 \\
\hline & Rogério Poloni & 1 \\
\hline & Marina Wöhlke Cyrillo & 1 \\
\hline & Geísa Schulze & 1 \\
\hline \multirow{4}{*}{ 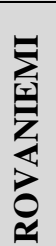 } & Anja Tuhoino & 3 \\
\hline & Sanna Kärkkäinen & 1 \\
\hline & Satu Luiro & 1 \\
\hline & Eila Linna & 1 \\
\hline
\end{tabular}

Em relação ao destino de Gramado constatou-se que todos entrevistados apresentam uma expectativa positiva em relação ao futuro do turismo $(n=4)$. Em especial, foi destacada a capacidade empreendedora da cidade, tanto na procura em atender as expectativas do turista como em se unir como comunidade para superar as dificuldades encontradas.

De uma forma geral, as expectativas futuras em relação ao turismo em Rovaniemi são consideradas como positivas. Percebe-se que os próprios desafios identificados pelos gestores públicos são encarados como grandes oportunidades para o desenvolvimento turístico do destino. A necessidade de um maior investimento em unidades hoteleiras e em resorts é apresentada como uma perspetiva de novos negócios para Rovaniemi. 


\section{Análise e Discussão dos Resultados da Pesquisa}

A partir das entrevistas tornou-se evidente a importância exercida pelo turismo para o desenvolvimento de ambos os destinos. Quer para Gramado quer para Rovaniemi, foi evidenciada uma dependência econômica em relação ao turismo. Em relação à inserção regional, ambos os destinos exercem um papel articulador para as políticas da Região em que estão inseridas, sendo que Rovaniemi se destaca nas entrevistas, por ser a capital administrativa da Região da Lapônia.

A participação da comunidade na elaboração das políticas do destino também é considerada significativa nos dois destinos. Em Gramado, apesar de se confirmar nas entrevistas que esta participação foi mais intensa no passado, ainda é um fator presente no processo de planejamento do destino. Em Rovaniemi constatou-se uma forte articulação regional com o envolvimento de todos os municípios integrantes da Região da Lapônia e uma significativa participação dos empresários no turismo, mostrando-se atuantes nas discussões locais.

Em relação às ações de marketing, as estratégias são distintas. Isto deve-se, principalmente, à configuração geográfica de cada cidade. Enquanto o destino brasileiro de Gramado se insere num contexto mais regional e de turismo doméstico, Rovaniemi desenvolveu-se como destino turístico internacional, focado nos mercados europeus e em países como a Grã-Bretanha e a Rússia. Neste sentido, as ações de marketing de Gramado centram-se em operadores e agentes turísticos nos mercados emissores do Brasil e da América Latina. Já Rovaniemi tem investido significamente na promoção do destino através das redes sociais e dos meios de comunicação digitais, sendo estas estratégias reforçadas nos planos estratégicos da cidade e da Região da Lapônia.

Considerando a interação entre os residentes e os visitantes, em ambos os destinos ficou evidente uma razoável harmonia nestas relações. Uma das evidências constatadas é de que a população tem plena consciência da importância do turista para a economia local. Em Gramado foi destacado o trabalho da consciencialização turística, realizado com as crianças nas escolas municipais. No destino de Rovaniemi, da mesma forma, os entrevistados destacaram como positiva a relação estabelecida entre o turista e o residente. Assim, o turismo é bem aceite em ambos os destinos. 
A mobilidade foi destacada como uma das grandes ameaças para os dois destinos, embora em contextos distintos. Enquanto em Gramado o ponto crítico está na mobilidade dentro do destino, impulsionado pela expansão urbana e pelo boom imobiliário, em Rovaniemi a preocupação central está na limitação dos vôos e conexões até ao destino. As mudanças climáticas também podem ser consideradas como uma ameaça para o destino, já que Rovaniemi tem como principal produto turístico o turismo de inverno (o gelo e a neve).

Quanto às perspetivas futuras para o turismo nos destinos, há tanto em Gramado quanto em Rovaniemi uma percepção positiva em relação ao futuro da atividade. Em Gramado foi salientada a capacidade empreendedora dos gramadenses em superar os desafios e tornar as ameaças em oportunidades. Da mesma forma, nas entrevistas realizadas com os gestores de Rovaniemi, ressalta-se que as ameaças também são encaradas como oportunidades para o destino. Em ambos os destinos foi destacada a necessidade de um maior investimento em empreendimentos hoteleiros. Isto demonstra um olhar positivo em relação ao crescimento turístico dos destinos pesquisados.

\section{Conclusões}

A partir da aplicação das entrevistas, foram encontradas semelhanças no percurso de desenvolvimento turístico de Gramado e de Rovaniemi. Uma das semelhanças é que ambos os destinos se desenvolveram a partir da temática Natal. Outro importante fator observado nas entrevistas foi a capacidade de integração e promoção regional dos destinos analisados. Tanto Gramado como Rovaniemi apresentaram-se como o destino propulsor do turismo da região em que se inserem. Nos dois destinos sobressaiu uma forte dependência econômica do turismo.

Em relação aos stakeholders, evidenciou-se, a partir das entrevistas, uma forte inter-relação entre os planejadores, gestores públicos e privados do turismo, organismos regionais e a população local, desempenhando papéis distintos no processo de desenvolvimento dos destinos. Conclui-se, assim, que a partir da metodologia utlizada à pesquisa foi possível estabelecer uma comparação das diferentes perceções de cada um dos participantes na entrevista. Nelas foi possível concluir que as atrações relacionadas ao tema Natal são o grande fator de desenvolvimento dos destinos pesquisados, refletido no forte comprometimento dos diferentes stakeholders para com o fortalecimento da marca "Natal". 
Cabe destacar que a pesquisa possui algumas limitações em relação à aplicação das entrevistas. Para tanto sugere-se um maior aprofundamento em relação a estes dados, especialmente no que se relaciona com a percepção sobre o turismo dos diferentes stakeholders, realizada em diferentes períodos. Tal análise permitirá uma maior lucidez em relação aos impactos desencadeados pelo turismo nas comunidades residentes. Neste sentido, além da aplicação de entrevistas com os atores envolvidos diretamente com a atividade turística, a abordagem junto aos residentes pode ser considerada uma estratégia que possibilite aproximar-se da efetiva prática das políticas públicas instituídas nos destinos.

\section{Referências}

BENVENISTE, Guy. Mastering the politics of planning: crafting credible plans and policies that make a difference. Jossey-Bass Inc Pub, 1989.

BERITELLI, Pietro. Cooperation among prominent actors in a tourist destination. Annals of Tourism Research, v. 38, n. 2, p. 607-629, 2011.

BRAMWELL, Bill; SHARMAN, Angela. Collaboration in local tourism policymaking. Annals of Tourism Research, v. 26, n. 2, p. 392-415, 1999.

BURNS, Peter M. Tourism planning: A third way?. Annals of Tourism Research, v. 31, n. 1, p. 24-43, 2004.

CITY OF ROVANIEMI. 2014. Rovaniemi Economic Development Programme: Objectives and Action Plan 2011-2016, Disponível em <http://www.rovaniemi.fi/loader.aspx?id = 301ffdf2-399e-46a2-9d9e32c7c034bcfc>. Acesso em 03 ago 2014.

COHEN, Louis; MANION, Lawrence; MORRISON, Keith. Research Methods in Education [5 th edn] London: Routledge Falmer. Teaching in Higher Education, v. 41, 2000.

COOPER, Christopher. "Progress in Tourism Research". In Cooper, C. (ed.), Classic reviews in tourism. Clevedon: Channel View Publications, p. 1-8, 2003.

DALONSO, Y. S. Avaliação de políticas públicas de desenvolvimento turístico das cidades de Gramado (Brasil) e Rovaniemi (Finlândia). Tese (Doutorado em Geografia). Instituto de Ciências Sociais. Universidade do Minho, Braga, Portugal. 327 p, 2015.

DALONSO, Yoná da Silva et al. O papel do Plano Diretor na constituição das políticas públicas para o turismo: um estudo da cidade de Gramado, Rio Grande do Sul (Brasil). 2012. Journal of Tourism and Development, 17,18(2), 909-923, 2012.

DREDGE, Dianne. Policy networks and the local organisation of tourism. Tourism Management, v. 27, n. 2, p. 269-280, 2006.

FINN, Mick; ELLIOTT-WHITE, Martin; WALTON, Mike. Tourism \& leisure research methods. Essex: Pearson Education Limited, 2000.

FRIEDMAN, Andrew L.; MILES, Samantha. Developing stakeholder theory. Journal of management studies, v. 39, n. 1, p. 1-21, 2002.

GETZ, Donald. Event studies: Theory, research and policy for planned events. Routledge, 2012. 
HAAHTI, Antti; YAVAS, Ugur. The effect of customer satisfaction with leisure services on behavioral intentions: A study of visitors to Santa Park in Lapland. Services Marketing Quarterly, v. 26, n. 3, p. 1$12,2005$.

HALL, Colin Michael. Tourism planning: policies, processes and relationships. Pearson Education, 2000.

HEALEY, Patsy. Collaborative planning in a stakeholder society. Town planning review, v. 69, n. 1, p. 1. 1998.

INSTITUTO BRASILEIRO DE GEOGRAFIA E ESTATÍSTICA - IBGE. 2013. Censo demográfico 2013. Disponível em: <http://www.ibge.gov.br/lojavirtual/fichatecnica.php?codigoproduto=90220.> Acesso em 28 jan. 2014.

JENNINGS, Gayle Ruth. Methodologies and methods. In Jamal T. e Robinson, M., The Sage handbook of tourism studies, London: Sage Publications, p. 672-692, 2009.

LAPPI. 2011. Lapland Tourism and Development Strategy. Disponível em: <http://matkailu.luc.fi/loader.aspx?id=1aacbbe8-cb33-46e0-a892-cece0b24a9b6>. Acesso em: 20 nov. 2014.

MALEK, Anahita; COSTA, Carlos. Integrating Communities into Tourism Planning Through Social Innovation. Tourism Planning \& Development, n. ahead-of-print, p. 1-19, 2014.

MINISTRY OF EMPLOYMENT AND THE ECONOMY OF FINLAND, MEE. 2014. Tourism's significance to Finland National Economy. Disponível em: < https://www.tem.fi/en/enterprises/tourism/tourism_s_significance_to_finland_s_national_economy. > . Acesso em 11 nov.2014.

MINISTÉRIO DO TURISMO. Estudo de Competitividade dos 65 Destinos Indutores do Desenvolvimento Turístico Regional - Relatório Brasil 2009. Brasília, 2009.

PRETES, Michael. Postmodern tourism: the Santa Claus Industry. Annals of Tourism Research, v. 22, n. 1, p. 1-15, 1995.

REJOWSKI, Miriam. Turismo e pesquisa científica. 3. ed. São Paulo: Paulo, 1999.

SAUTTER, Elise Truly; LEISEN, Brigit. Managing stakeholders a tourism planning model. Annals of Tourism Research, v. 26, n. 2, p. 312-328, 1999.

SECRETARIA DE TURISMO DE GRAMADO. 2013. Natal Luz de Gramado recebe 1,5 milhão de pessoas. Disponível em: http://www.gramado.rs.gov.br/index.php/Turismo/-Natal -Luz-de-Gramadorecebe-1,5-milhoes-de-Pessoas-em-2012.html. Acesso em: 28 jan. 2013.

TERVO-KANKARE, Kaarina; HALL, C. Michael; SAARINEN, Jarkko. Christmas tourists' perceptions to climate change in Rovaniemi, Finland. Tourism Geographies, v. 15, n. 2, p. 292-317. 2013.

VERNON, Jon et al. Collaborative policymaking: Local sustainable projects. Annals of Tourism Research, v. 32, n. 2, p. 325-345, 2005.

WALIGO, Victoria M.; CLARKE, Jackie; HAWKINS, Rebecca. Implementing sustainable tourism: A multi-stakeholder involvement management framework. Tourism Management, v. 36, p. 342-353, 2013.

YÜKSEL, Fisun; BRAMWELL, Bill; YÜKSEL, Atila. Centralized and decentralized tourism governance in Turkey. Annals of Tourism Research, v. 32, n. 4, p. 859-886, 2005.

\section{Recebido em: 15/06/2015 ( $1^{\text {a }}$ versão) $28 / 07 / 2015$ ( $2^{a}$ versão) \\ Aprovado em: 08/08/2015}

